\title{
Efficacy of EMG/Bioimpedance-Triggered Functional Electrical Stimulation on Swallowing Performance
}

\author{
Corinna Schultheiss (1), Thomas Schauer (2), Holger Nahrstaedt (2), Rainer O. Seidl (1), \\ Jehoschua Bieler (1)
}

\author{
(1) Unfallkrankenhaus, Ear, Nose, and Throat Unit, Berlin, Germany; (2) Control Systems \\ Group, Technische Universität, Berlin, Germany
}

This article is distributed under the terms of the Creative Commons Attribution Noncommercial License (CC BY-NC 4.0) which
permits any noncommercial use, distribution, and reproduction in any medium, provided the original author(s) and source are credited.

\begin{abstract}
In order to support swallowing, the efficacy of functional electrical stimulation for different stimulation settings of the submental musculature has been investigated. The stimulation was administrated at rest and synchronously to voluntary initiated swallows. The onset of a swallow was detected in real-time by a combined electromyography/ bioimpedance measurement at the neck in order to trigger the stimulation. The amplitude and speed of larynx elevation caused by the FES has been assessed by the observed change in bioimpedance whereas a reduction of bioimpedance corresponds to an increase in larynx elevation. Study results from 40 healthy subjects revealed that $73 \%$ of the subjects achieved a larger and faster larynx elevation during swallowing with triggered FES and therefor a better protection of their airways. However, we also observed a decrease in larynx elevation compared to normal swallowing in 11 out of the 40 subjects what might not benefit from such a treatment. The largest improvement of larynx elevation and speed during swallowing could be achieved with three stimulation channels formed by four electrodes in the submental region.
\end{abstract}

Key Words: triggered functional electrical stimulation, dysphagia, rehabilitation, bioimpedance, electromyography

Eur J Transl Myol 26 (4): 283-286

Electrical stimulation has been widely applied in the therapy of patients with dysphagia. One general aim is to train the submental and suprahyoidal and infrahyoidal muscles by applying electrical stimulation for longer periods of time by means of surface electrodes. ${ }^{1,2}$ Most existing systems do not take into account present volitional swallowing activity of the patients. As reported $\mathrm{in}^{3}$, the stimulation in the vicinity of larynx can even have a negative effect on the protection of the airways during a real swallow as muscle can be activated that lower the larynx (e.g. the M. sternohyoideus). Only stimulation of the submental muscles proved to be safe with a positive effect on swallowing mechanics most of the time ${ }^{3}$. Another therapy approach is to apply sensory stimulation to the posterior pharyngeal wall by using a stimulation catheter that is inserted via the nasal passage ${ }^{4}$. Also in the case, stimulation is applied in a non-controlled manner.

In our previous work ${ }^{5}$ we demonstrated that a functional electrical stimulation of the submental muscles synchronously to the voluntary induced swallows is feasible and can help in increasing the amount and velocity of larynx elevation. The onset of stimulation was detected in real-time by an initial drop in bioimpedance (BI) in conjunction with EMG activity measured at the neck. The bioimpedance measurement (absolute value of bioimpedance measured at $50 \mathrm{kHz}$ ) was furthermore used to assess the resulting larynx elevation (negative proportional relation) during swallowing $^{6}$. As alternative to our automatic triggering, a hand switch triggered stimulation can be used to synchronize FES with swallows ${ }^{7}$.

In this contribution, we evaluate the effect of different submental stimulation strategies (electrode configurations) and two different stimulation intensities on the bioimpedance and therefore on the larynx elevation.

\section{Materials and Methods}

The examination was performed on 40 healthy subjects (18 females, 22 males, mean age of 29 years) without any swallowing impairment. The study was approved by the Charite Berlin Ethics Committee in the vote EA1/019/10.

The subjects were stimulated at rest (non-swallowing) and during swallowing water using an adapted current- 


\section{EMG/Bioimpedance-Triggered FES for Swallowing}

Eur J Transl Myol 26 (4): 283-286

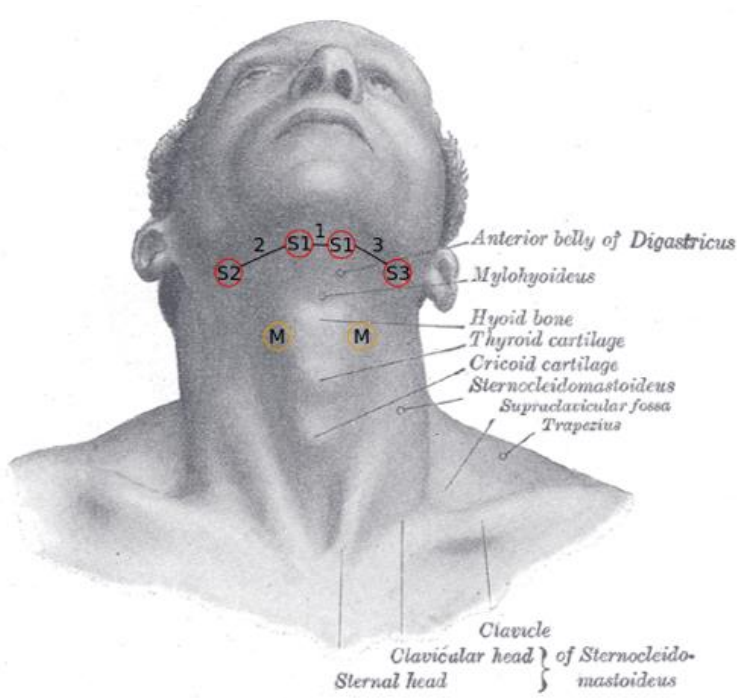

Fig 1. Position of electrodes for forming three stimulation channels. $M-$ measurement electrodes, S1-S3 - stimulation electrodes, 1 - 3 channels.

Modified from:https://commons. wikimedia.org /wiki/ File:Gray1195.png

controlled stimulator (RehaStim1, HASOMED GmbH) with a maximum current amplitude of $30 \mathrm{~mA}$. For the onset detection of the water swallows we used a selfdeveloped EMG/BI measurement system ${ }^{6}$.

Four self-adhesive surface electrodes (MultiStick-Gel, 25mm, Axelgaard, Denmark) were attached for FES at the submental region. We placed two electrodes $(2 \mathrm{xS} 1)$ on the mouth base and two other electrodes in the yaw angle (S2 and S3). Based on these four electrodes three stimulations channels have been set up (cf. Fig. 1): Channel 1 (S1-S1), Channel 2 (S1-S2) and Channel 3 (S1-S3). Three stimulation settings were applied:

A) stimulation via channel 1 only,

B) stimulation via channels 2 and 3 (sequentially with $1.5 \mathrm{~ms}$ interval between the two channels),

C) stimulation on all three channels 1, 2 and 3 (sequentially with $1.5 \mathrm{~ms}$ interval between the three channels).

The stimulation intensity was set to $30 \mathrm{~Hz}$ for all settings. The pulse width of the biphasic stimulation

Table 1. Average maximally tolerated stimulation intensities for the different stimulation settings: A) Channel 1, B) Channel $2 \& 3$, C) Channel 1, 2, \& 3

\begin{tabular}{lcccccc}
\hline & \multicolumn{2}{c}{$\begin{array}{c}\text { Initial intensity } \\
\boldsymbol{I}_{\text {init }}(\mathbf{m A})\end{array}$} & \multicolumn{3}{c}{$\begin{array}{c}\text { Updated intensity } \\
\boldsymbol{I}_{\text {update }}(\mathbf{m A})\end{array}$} \\
\hline Setting & $\boldsymbol{A}$ & $\boldsymbol{B}$ & $\boldsymbol{C}$ & $\boldsymbol{A}$ & $\boldsymbol{B}$ & $\boldsymbol{C}$ \\
Total & 8 & 7 & 7 & 11 & 9 & 10 \\
Male & 8 & 7 & 8 & 12 & 10 & 10 \\
Female & 7 & 6 & 7 & 10 & 9 & 9 \\
\hline
\end{tabular}

Table 2. Effect of stimulation intensity on BI drop (larynx elevation) at rest (for nonswallows). Reported are mean values with standard deviations

\begin{tabular}{lcc}
\hline Parameters of BI drop & $\boldsymbol{I}_{\text {init-Intensity }}$ & $\boldsymbol{I}_{\text {update-Intensity }}$ \\
\hline Amplitude $(\mathbf{O h m})$ & $0.186(0.14)$ & $0.278(0.19)$ \\
Speed $(\mathbf{O h m} / \mathbf{s})$ & $1.967(10.06)$ & $1.675(0.27)$ \\
\hline
\end{tabular}

pulses was fixed at $200 \mu \mathrm{s}$, whereas the current amplitude $I$ served as adjusttable stimulation intensity. Stimulation with each setting was repeated three times for each subject in a fixed order (at rest and during swallowing). Additionally, swallows without stimulation have been recorded. Initially, before the first measurement (first application of the three stimulation settings), we determined the maximally tolerated stimulation intensity for each setting in every subject and denoted this values as $I_{\text {init }}$. The obtained values were used for the first application of the stimulation settings. One readjustment of these values took place after the first measurement round was completed and before applying all stimulation settings another two times. The updated intensities were denoted as $I_{\text {update }}$. In order to assess the effect of stimulation at rest (non-swallows) and to compare swallows without and with stimulation, the amplitude $A_{B I}$ of the bioimpedance drop (positive values for a drop in $\mathrm{BI})$ and the corresponding speed $S_{B I}\left(A_{B I}\right.$ divided by the time to reach the maximum drop) have been determined for stimulation at rest and during all swallows. A larger amplitude $A_{B I}$ corresponds to a larger larynx elevation, while the elevation velocity is directly described by the speed of the BI change 6 . A Wilcoxon test has been applied to look for statistically significant differences in the stimulation settings.

\section{Results}

The mean $I_{\text {init }}$-intensity was $8 \mathrm{~mA}$ (male $=8 \mathrm{~mA}$, female $=7 \mathrm{~mA}$ ). The mean $I_{\text {update-intensity was } 10 \mathrm{~mA}}$ $($ male $=10 \mathrm{~mA}$, female $=9 \mathrm{~mA})$. Details are reported in Table 1.

Table 3. Comparison of swallows with and without stimulation for different intensities. Reported are mean values with standard deviations of the BI drop amplitude (larynx elevation) and speed (larynx velocity) for 29 out of the 40 patients who showed an increase in larynx elevation compared to unassisted swallowing.

\begin{tabular}{lccc}
\hline \multicolumn{1}{c}{$\begin{array}{c}\text { Parameters } \\
\text { of BI drop }\end{array}$} & $\begin{array}{c}\text { Swallow } \\
\text { without } \\
\text { stimulation }\end{array}$ & $\begin{array}{c}\text { Swallow with } \\
\text { stimulation } \\
\left(\boldsymbol{I}_{\text {init }}\right)\end{array}$ & $\begin{array}{c}\text { Swallow with } \\
\text { stimulation } \\
\left(\mathbf{I}_{\text {update }}\right)\end{array}$ \\
\hline $\begin{array}{l}\text { Amplitude } \\
(\mathbf{O h m})\end{array}$ & $0.637(0.28)$ & $0.787(0.34)$ & $0.845(0.36)$ \\
Speed $(\mathbf{O h m} / \mathbf{s})$ & $2.735(1.38)$ & $3.175(1.34)$ & $3.676(1.75)$ \\
\hline
\end{tabular}




\section{EMG/Bioimpedance-Triggered FES for Swallowing}

Eur J Transl Myol 26 (4): 283-286

Table 4. Comparison of swallows with and without stimulation for different intensities. Reported are mean values with standard deviations of the BI drop amplitude (larynx elevation) and speed (larynx velocity) for 11 out of the 40 patients who showed a decrease in larynx elevation compared to unassisted swallowing.

\begin{tabular}{cccc}
\hline $\begin{array}{c}\text { Parameters } \\
\text { of BI drop }\end{array}$ & $\begin{array}{c}\text { Swallow } \\
\text { without } \\
\text { stimulation }\end{array}$ & $\begin{array}{c}\text { Swallow } \\
\text { with } \\
\text { stimulation } \\
\left(\boldsymbol{I}_{\text {init }}\right)\end{array}$ & $\begin{array}{c}\text { Swallow } \\
\text { with } \\
\text { stimulation } \\
\left(\mathbf{I}_{\text {update }}\right)\end{array}$ \\
\hline $\begin{array}{c}\text { Amplitude } \\
(\mathbf{O h m})\end{array}$ & $0.664(0.30)$ & $0.588(0.26)$ & $0.571(0.28)$ \\
Speed $(\mathbf{O h m} / \mathbf{s})$ & $2.486(0,99)$ & $2.691(1.44)$ & $2.526(1.49)$ \\
\hline
\end{tabular}

The evaluation included 598 non-swallows. For the nonswallows we could prove significant differences ( $\mathrm{p}<$ $.001)$ between the $I_{\text {init }}-$ intensity $(\mathrm{n}=279)$ and the $I_{\text {update }}{ }^{-}$ intensity $(\mathrm{n}=319)$. For the amplitude $A_{B I}$, the highest value was detected for the $I_{\text {update }}$-intensity. However, the speed shows the highest value with the $I_{\text {init-intensity (see }}$ Table 2). We observed an increase of $25 \%$ in the maximally tolerated stimulation intensity by updating this value.

Overall, we included 2132 swallows (729 swallows without stimulation, 1403 swallows with stimulation) in our analysis. For 29/40 (520 swallows without stimulation, 1028 swallows with stimulation) subjects we detected a significant increase $(\mathrm{p}<.001)$ in the amplitude $A_{B I}$ and speed $S_{B I}$ of the BI drop (i.e. laryngeal elevation) when comparing FES-assisted swallowing and normal swallowing. The highest values were detected for $I_{\text {update. }}$ Table 3 shows the obtained results for this subgroup of subjects. The corresponding 29/40 subjects were 12 females and 17 males.

All the remaining 11/40 subjects showed a significant decrease of the amplitude $\left(\mathrm{p}=.003^{* *}\right)$ under swallowing with stimulation compared to unassisted swallowing. The speed of laryngeal elevation shows no significant variance (see Table 4). The 11/40 subjects who showed a decrease of swallowing performance were 6 females and 5 males.

For the two sub groups of subjects (29/40 and 11/40) reported above we investigated again the effect of

Table 5. Effect of stimulation intensity on BI drop (larynx elevation) at rest (for non-swallows) for the 29/40 subjects who showed an increase in larynx elevation by FES during swallwing compared to normal swallowing. Reported are mean values with standard deviations.

\begin{tabular}{lcc}
\hline Parameters of BI drop & $\boldsymbol{I}_{\text {init-Intensity }}$ & $\boldsymbol{I}_{\text {update-Intensity }}$ \\
\hline Amplitude $(\mathbf{O h m})$ & $0.195(0.15)$ & $0.297(0.19)$ \\
Speed $(\mathbf{O h m} / \mathbf{s})$ & $1.088(0.94)$ & $1.622(1.10)$
\end{tabular}

Table 6. Effect of stimulation intensity on BI drop (larynx elevation) at rest (for nonswallows) for the 11/40 subjects who did not show an increase in larynx elevation by FES during swallowing compared to normal swallowing. Reported are mean values with standard deviations.

\begin{tabular}{lcc}
\hline Parameters of BI drop & $\boldsymbol{I}_{\text {init.-Intensity }}$ & $\boldsymbol{I}_{\text {update }}$-Intensity \\
\hline Amplitude $(\mathbf{O h m})$ & $0.149(0.12)$ & $0.221(0.17)$ \\
Speed $(\mathbf{O h m} / \mathbf{s})$ & $0.936(1.09)$ & $1.305(1.24)$ \\
\hline
\end{tabular}

stimulation at rest in order to evaluate if a prediction of the responses during FES-assisted swallowing based on the stimulation results at rest is possible. The obtained data are displayed in the Tables 5 and 6 . For the 29/40 sub group the differences between the $I_{\text {init }}$ and $I_{\text {update }}$ were significant for $A_{B I}(\mathrm{p}<.001)$ and $S_{B I}(\mathrm{p}$ $<.001)$. The highest value for $A_{B I}$ and $S_{B I}$ were shown again with the $I_{\text {update }}$-intensity. For $11 / 40$ sub group the differences between the $I_{\text {init }}$ and $I_{\text {update }}$ were significant for $A_{B I}\left(\mathrm{p}=.007^{* *}\right)$ and $S_{B I}\left(\mathrm{p}=.005^{* *}\right)$. As shown for the 29/40 subjects the highest values for $11 / 40$ subjects were also with the $I_{\text {update }}$. Both sub groups showed an increase in larynx elevation at rest by applying electrical stimulation.

Bioimpedance, i.e. larynx elevation and speed, were influenced differently by the three stimulation settings. For stimulation at rest (non-swallows), the amplitude $A_{B I}$ shows the highest value for the stimulation setting $A$ (channel 1) and the lowest value for the stimulation setting B (channel $2 \& 3)\left(\mathrm{p}=, 013^{*}\right.$ (A vs B)). The parameter speed $S_{B I}$ shows the highest value for stimulation setting $C$ (channel $1,2 \& 3$ ) and the lowest for setting B (channel $2 \& 3)\left(\mathrm{p}=.015^{*}(\mathrm{C}\right.$ vs B)) (cf. Table 7).

During swallowing with FES support, we found significant differences in the stimulation settings for $A_{B I}$ ( $\mathrm{p}<.001$ (A vs C), $\mathrm{p}=.035^{*}$ (B vs C)), and for $S_{B I}$ $\left(\mathrm{p}=.043^{*}\right.$ (B vs $\left.\mathrm{C}\right)$ ). The highest values for amplitude and speed were detected for setting $\mathrm{C}$ (cf. Table 8).

Table 7. Influence of the stimulation setting on the parameters of the BI drop during rest. Reported are mean values with standard deviations for all 40 subjects and both stimulation intensities.

\begin{tabular}{lccc}
\hline $\begin{array}{l}\text { Parameters } \\
\text { of BI drop }\end{array}$ & Setting A & Setting B & Setting C \\
\hline Amplitude & 0.254 & 0.213 & 0.242 \\
$($ Ohm) & $(0.18)$ & $(0.16)$ & $(0.18)$ \\
Speed & 1.829 & 1.590 & 2.025 \\
$($ Ohm/s) & $(10.03)$ & $(3.06)$ & $(7.08)$ \\
\hline
\end{tabular}




\section{EMG/Bioimpedance-Triggered FES for Swallowing}

Eur J Transl Myol 26 (4): 283-286

Table 8. Influence of the stimulation setting on the parameters of the BI drop during swallowing with FES support. Reported are mean values with standard deviations for all 40 subjects and both stimulation intensities.

\begin{tabular}{lccc}
\hline $\begin{array}{c}\text { Parameters } \\
\text { of BI drop }\end{array}$ & Setting A & Setting B & Setting C \\
\hline Amplitude & 0.746 & 0.718 & 0.774 \\
$($ Ohm) & $(0.36)$ & $(0.34)$ & $(0.36)$ \\
Speed & 2.430 & 2.914 & 3.391 \\
$(\mathbf{O h m} / \mathbf{s})$ & $(6.50)$ & $(3.77)$ & $(2.02)$ \\
\hline
\end{tabular}

\section{Discussion}

The study results show that an EMG/BI-triggered FES is feasible in a larger population of healthy subjects. Subjects got used to FES at the submental region, and maximally tolerated stimulation intensities could be increased by $25 \%$ after some stimulations. The effect of FES on the amplitude of the BI drop (the larynx elevation) could be modulated by the stimulation intensity. For EMG/BI-triggered FES in dysphagia therapy, a four electrode setup can be recommended as that gives the largest support in larynx elevation (in terms of amplitude and speed). Based on the four electrodes three stimulation channels are formed.

$73 \%$ of the subjects could significantly improve amplitude and speed of larynx elevation by EMG/BItriggered FES compared to normal water swallowing without FES. In patients this will potentially lead to improve of protection of the airways. However, we also found a decrease in larynx elevation in $27 \%$ of the healthy subjects. Such an undesired outcome will be automatically detected by the EMG/BI measurement system during swallowing. Patients who show a decrease in larynx elevation are probably no candidates for such a triggered FES approach.

A simple prediction of the effect of FES during swallowing on the results obtained by stimulation at rest was not possible. During stimulation at rest we always observed an increase by larynx elevation by applying electrical stimulation to the submental muscles. We are currently investigating possible reasons why some subjects have negative responses during FES-assisted swallowing while showing positive responses at rest.

After we have found the best stimulation setting, we plan a larger study with patients suffering from dysphagia to confirm these results with patients.

\section{Contributions}

CS: study design, statistical analysis, manuscript writing; TS: study design, manuscript writing;HN: development of BI/EMG-triggered stimulation; ROS: study design;
JB: data collection and interpretation; study design, manuscript writing; All authors: manuscript reviewing.

\section{Acknowledgement}

This work was partly funded by the Federal Ministry for Economic Affairs and Energy (BMWi) within the ZIM project MultiEMBI (KF 2392314CS4).

\section{Conflict of Interest}

The authors declare no potential conflict of interests.

\section{Corresponding Author}

Corinna Schultheiss, Warenerstr. 7, D-12683 Berlin +493031423680

E-mail: corinnaschultheiss@gmail.com

E-mails of coAuthors

Thomas Schauer: Schauer@control.TU-Berlin.de Holger Nahrstaedt: Nahrstaedt@ control.TU-Berlin.de Rainer O. Seidl: Rainer.Seidl@ukb.de Jehoschua Bieler: Jehoschua.Bieler@ukb.de

\section{References}

1. Freed M L, Freed L, Chatbrn R L, Christian M. Electrical stimulation for swallowing disorders caused by stroke. Respir Care. 2001;46:466-74.

2. Seidl R O, Nahrstaedt H, Schauer T. Electric stimulation in dysphagia therapy - a review. Laryngorhinootology. 2009;88:768-74.

3. Ludlow C L, Humbert I, Saxon K, Poletto C, Sonies B, Crujido L. Effects of surface electrical stimulation both at rest and during swallowing in chronic pharyngeal Dasphagia. Dysphagia 2007;22:1-10.

4. Jayasekeran V, Singh S, Tyrrell P, Michou E, Jefferson S, Mistry S, Gamble E, Rothwell J, Thompson D, Hamdy S. Adjunctive functional pharyngeal electrical stimulation reverses swallowing disability after brain lesions. Gastroenterology 2010;138:1737-47.

5. Nahrstaedt H, Schultheiss $C$, Schauer T, and Seidl R O. Bioimpedance- and EMG-Triggered FES for Improved Protection of the Airway During Swallowing. Biomedical Engineering / Biomedizinische Technik. 2013; DOI: 10.1515/bmt-2013-4025.

6. Schultheiss C, Schauer T, Nahrstaedt H, and Seidl $\mathrm{R} O$. Evaluation of an EMG bioimpedance measurement system for recording and analysing the pharyngeal phase of swallowing. Eur Arch Otorhinolaryngol 2013;270 2149-56.

7. Burnett T A, Mann E A, Stoklosa J B, and Ludlow C L. Self-triggered functional electrical stimulation during swallowing. J Neurophysiol 2005;94:4011-18. 